\title{
Oblivious Parallel Probabilistic Channel Utilization without Control Channels
}

\author{
Christian Schindelhauer* \\ University of Paderborn \\ Heinz Nixdorf Institute \\ Fuerstenallee 11, 33102 Paderborn \\ schindel@upb.de
}

\author{
Kerstin Voß \\ University of Paderborn \\ Paderborn Center for Parallel Computing \\ Fuerstenallee 11, 33102 Paderborn \\ kerstinv@upb.de
}

\begin{abstract}
The research interest in sensor nets is still growing because they simplify data acquisition in many applications. If hardware resources are very sparse, routing algorithms cannot use data gathering. However, if a large number of channels can be used, then parallel transmission can compensate this drawback. If the senders and receivers are not known in advance, then a control channel poses a bottleneck for communication. We present an oblivious MAC protocol, called the Funnel protocol, where the channels are nearly optimally utilized in parallel. In this, senders and receivers choose for a polylogarithmic number of rounds (several sending attempts) a decreasing number of channels which are selected equiprobably.

Then, we show that a previously presented approach using only one round and therefore one type of probability distribution is optimal up to some constant factor, and considerably worse than the Funnel protocol. The protocol works with few resources if an sufficient number of channels is available. The Funnel protocol is simple, elegant, and does not need to know the number of senders and receivers, thus being oblivious.

On the bottom line we prove that small messages can be efficiently transmitted by the MAC layer in parallel without a control channel if more than one channel for communication can be used.
\end{abstract}

\section{Introduction}

Sensor nets have many undisputed fields of applications. Nodes collect sensory attributes and send these to a cen-

\footnotetext{
*Partially supported by the DFG-Sonderforschungsbereich 376 and by the EU within 6th Framework Programme under contract 001907 "Dynamically Evolving, Large Scale Information Systems” (DELIS).
}

tral station $\left[\mathrm{SCI}^{+} 01\right]$. Their hardware consists of a central processing unit (CPU), storage (RAM and FLASH), sensor, radio transceiver, and an autarkic energy source. These resources are very limited because the nodes have to be small and inexpensive [WLLP01, $\left.\mathrm{HSW}^{+} 00\right]$. Accordingly, algorithms need to be time and memory efficient. So special algorithms for sensor networks have to be developed.

In wireless sensor nets the range for sending and receiving is very limited because nodes run on batteries. In order to establish the communication between every node and the central base, multi-hop connections are built. For this reason, the tasks of nodes are not restricted to collecting data. Message forwarding is also obligatory. Rather nodes should forward messages by a flexible choice of neighbors because of network dynamics and occurrence of radio interference in communication.

A paradigm in routing protocols is to use one of the supported frequencies of the radio transceiver. However, the state-of-the-art radio transceivers support more than one frequency. Medium access protocols should take advantage of these plurality to increase the message throughput by parallel transmissions. For example, the Chipcon CC1000 supports at most 13 frequencies of $64 \mathrm{kHz}$ if the safety section is as large as the interval for a channel [JE03]. Additionally, if messages are very short, which implies short transmission times, even more frequencies can be simulated by using TDMA (time division multiple access). With TDMA, nodes inactively wait until the correct time slot for the sending or receiving attempt. Since, nodes typically consume most energy for sending and receiving actions [LH05], the energy consumption for inactive rounds is only little and can be neglected.

The task of channel selection and utilization of the communication medium is the classical problem of the Medium Access Layer which is positioned in the Data Link Layer in the OSI Model (see IEEE P802.3ae). Using an uni- 
form probability distribution for selecting the communication channel (for sending or receiving actions) performs best if the number of senders is equivalently to the number of channels [SV05]. Unfortunately, the number of senders and receivers for each sending attempt depends on many factors (number of nodes in sending range, probability for a message, probability of failure in previous attempts ...). Resulting, it cannot be estimated very well.

\subsection{The Problem}

The Funnel Protocol is a MAC-protocol for a vast number of nodes which can be classified as senders and receivers. Any receiver is eligible for transporting any sender's message. We assume for the theoretical analysis that all nodes are within the same sending and receiving range and the communication is symmetric. Furthermore, all nodes have access to the same number of channels which can be implemented by different frequencies (FDMA - Frequency Division Multiple Access), different encodings (CDMA Code Division Multiple Access), or even time slots (TDMA - Time Division Multiple Access). With these techniques the number of channels will be larger than the number of senders and of receivers. We assume that adequate multiple access protocols are in place and work on all nodes which implies the existence of a synchronization mechanism.

This paper discusses how to use the uniform distribution in the MAC layer in order to maximize the number of successful delivered messages within several parallel sending attempts, i.e. multiple rounds. A theoretic analysis of the node communication in such a multiple round scheme is presented. Because of the Funnel protocol's randomized nature and its simplicity we also expect positive results in a more realistic scenario with restricted transmission lengths as well as with hidden and exposed terminal problems. We leave this question open for further research. Recent experiments show that this assumption does not impair the practicality of this protocol.

All sender nodes randomly selects a channel from a large set of $C$ channels and each sender sends a message. At the same time each of the receivers randomly selects a channel. Now, we have two models for a successful transmission:

\section{one-to-many}

A message transmission is successful if and only if one sender and at least one receiver have chosen the same channel. Then, every receiver takes and acknowledges the message. Resultant from the received acknowledgement the sender drops out of the set of senders. The receivers become senders until they have successfully forward the message. Since more than one receiver may forward one message, duplicates may occur.

\section{one-to-one}

Only if one sender and exactly one receiver have chosen the same channel, a message is transmitted. An acknowledgments on the same channel ensures that the nodes change their roles, i.e. the sender becomes a receiver if it has no further message and the receiver becomes a sender in the later rounds since it has to forward the message.

Although both approaches seem to give up too early in the well investigated case of classical medium access to a single channel, we see that even under these restricted communication models fast and reliable parallel transmission can be achieved.

We emphasize the fact that the initial number of senders $n$ and the number of receivers $m$ are not known. The only assumption we rely upon is $n, m \leq C$. The optimization objective of the routing protocol is to minimize the number of rounds which are required to successfully forward (with a high probability) all messages. This minimization results from the idea of reducing consumed energy. So, the Funnel protocol is described by the choice of the probability distributions used from senders and receivers in each round. The quality of the distribution selection is measured by the expected value of the number of transmitted messages. In this paper we present a simple and oblivious method which works for nearly any resource restrictions.

The structure of this paper is as following: At first, related work is presented. Afterwards the explicit model is described. Section 4 presents and analyzes the Funnel protocol, the main result of this paper. Also the open question of [SV05] is solved here: whether the $s$-factorized geometric probability distribution is optimal for the single round model. In the last section a conclusion is given.

\section{Related Work}

The presented related work focuses on routing and MAC protocols because both are responsible for a good message throughput. Since nodes have only sparse resources, special routing algorithms were developed for sensor networks. At the first look sensor networks and mobile ad-hoc networks have many similarities. However, since restrictions in developing algorithms for mobile ad-hoc networks do not suffice the few resources of sensor nodes, established approaches (proactive and reactive) are rarely realized in sensor networks. Anyhow, as a reactive method for sensor networks we mention the Pulse Protocol [AHR04a, $\mathrm{AHR}^{+}$04b].

There exists protocols using data gathering, for example [HLMV05], the LEACH Protocol [HCB00], and the Data Collection Protocol (DCP) [HGT04]. The method of data 
gathering has certainly many advantages but it cannot be used with every node specification (if the nodes have only little RAM, e.g. 256 Byte). In our work we assume that nodes possess too less storage for data aggregation in order to make no resource restrictions. Since the developed Funnel protocol is independent from data aggregation, it's method can also be integrated in protocols with data gathering.

Further differences in routing protocols exist in different communication directions. Most protocols consider communication from sensor nodes with a central station. According to their primary task (deliver sensor information to the central station) we also assume this many-to-one routing. Furthermore, protocols for communication between several sensor nodes were developed, e.g. the Direct Diffusion Mechanism [HCB00, IGE00].

An important aspect in many-to-one routing protocols is fairness. If many sensor nodes want to communicate with one central station, all nodes should have the same throughput and latency in message delivering. In [EB04] the routing fairness is achieved by an end-to-end congestion protocol: Root nodes inform their children from which nodes they have already transmitted too many packages. So the children can temporarily reduce the transmission rates of those nodes.

An additional precondition for our work is that nodes are clock-synchronized. Synchronization minimizes energy consumptions by preventing effectless sending or listening on a channel. Synchronization methods for traditional distributed systems cannot be utilized in sensor networks. On the one hand the resources are too restricted, on the other hand the synchronization precision does not suffice. Accordingly, special synchronization methods have been developed for sensor networks: If nodes communicate wireless, the messages are broadcasted. Accordingly, the Reference Broadcast Synchronization can be used for clock time adjustment [EGE02]. An improvement of this protocol is the Timing-Sync Protocol for Sensor Networks [GKS03]. For example, it achieves a higher precision. In sensor networks with sporadic communication the Improved IntervalBased Clock-Synchronization can be used [BMT04]. This method is worst-case-optimal by taking advantage of the typical drift diversity of the nodes' clocks.

The synchronization makes a coordinated awake possible. Several MAC protocols have already been developed with this feature. An energy-efficient protocol is the time-slotted PMAC [ZRS05] because the duration of sleeping depends on the traffic in the net. If there is no traffic, the nodes sleep longer to save energy. The sleep-wake-up pattern can be used in nearly every protocol and is also applicable to the channel selection method presented here.

One of the main tasks of the MAC layer is to deal with collisions. To avoid interference caused by sending of dif- ferent nodes, senders and receivers can arrange data transfer with several control messages. That way of medium access is realized, for example, in PAMA [SR98] and MACA [Kar90, Sch00]. Also, TRAMA [ROGLA03] has a schedule-based mechanism to avoid collisions. A mentionable characteristic of this protocol is its support of unicast, multicast, and broadcast traffic. Additionally, nodes are switched dynamically to a low power mode depending on the current traffic pattern. This feature is an improvement of the static method in S-MAC [YHE04]. Another approach of saving energy is realized in [KKIK03] by balancing the energy consumption over all nodes. That means, nodes which have less energy than others sleep longer to save energy. The S-MAC protocol was also improved in BMAC [PHC04] which is characterized by its flexibility and on-the-fly reconfiguration for optimizing performance.

In collision-free protocols, control messages do not always create a negligible overhead. If the transmitted messages are very small, the overhead is relatively large and omitting control messages can be desirable $\left[\mathrm{SJR}^{+} 04\right]$. This idea of allowing interference was already integrated in ALOHA [Rob75]: In ALOHA nodes send their messages at any time. When collisions have appeared, nodes repeat the packages after a randomized waiting-time. As a variant we mention the slotted ALOHA in which nodes are allowed to send exclusively in special defined slots [VL90, Nam84, BZ97]. There, the slotted ALOHA is discussed in different types of networks (mobile and cellular systems).

Avoidance of interference can be achieved by multiplexing, one of the tasks of the MAC layer [Sch00]. There are four dimensions of multiplexing: space, time, frequency, and code. Frequency division multiplexing (FDM) is integrated in the developed Funnel protocol because the used frequency band is divided in several channels. Additionally, if the hardware does not support as many channels as needed, it is advantageous to use time division multiplexing (TDM). Then with the aid of internal rounds, TDM simulates more channels than the hardware supports. However, messages can still collide without access control.

It is a paradigm in sensor networks to use one channel for communication. In [SV05] we have already presented a new concept in which one of several frequencies is probabilistic selected. There, the expected number of delivered messages in one sending attempt was optimized. In this work we want to minimize the error rate of unsuccessful transmissions in a multiple round scheme while keeping the number of rounds low.

We argue in favor of utilizing many frequencies because it increases throughput. Higher throughput leads to energy savings and a longer lifetime of the nodes. This is our motivation for discussing the integration of this feature into the MAC layer.

The channel selection in the developed Funnel protocol is 
completely oblivious and random. There exists no control channel for arranging meeting on another channel. Also, nodes do not work on a pseudo-random channel hopping like in Bluetooth [Gro99, Gol04]. There, in every piconet clients follow the pattern of the master. This method was also transferred for smart devices with Bluetooth like special sensor nodes in [SR03].

\section{The Model}

The significant measure for the throughput is the expected value of successfully transmitted messages in one sending phase. We are aware that in the one-to-many model many receivers can receive the same message. Following, message duplicates can arise (see Fig. 3). This problem, however, does not occur in the one-to-one round model and we neglect its impact in the one-to-many protocol. Further research will prove whether the number of such message duplicates is of significance.

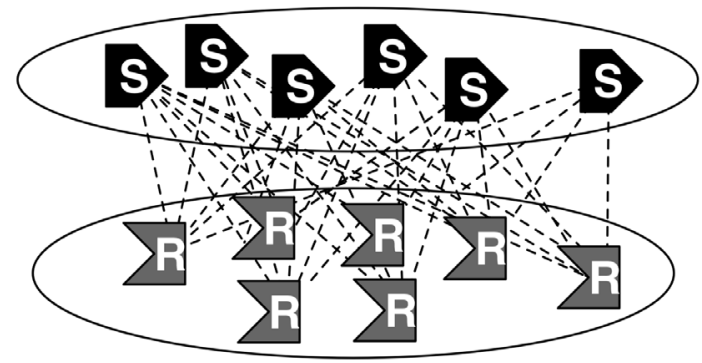

Figure 1. The sender/receiver situation

Definition 1 Given $n$ senders, $m$ receivers, and $C$ channels. Each sender selects channel $i \in C$ independently with probability $p_{i}$ where each receiver selects this channel with probability $p_{i}^{\prime}$. Define the random variable $M_{p, p^{\prime}}^{1-m}(n, m)$ as the number of forwarded messages in the one-to-many model and let $M_{p, p^{\prime}}^{1-1}(n, m)$ be the one for the one-to-one model.

If $p=p^{\prime}$, we denote $M_{p}^{1-1}(n, m):=M_{p, p^{\prime}}^{1-1}(n, m)$. Furthermore if each of the $C$ channels is chosen with the same probability $p_{i}=\frac{1}{C}$ (in the case of the uniform distribution), we simplify to $M_{1 / C}^{1-1}(n, m)$. Equivalent notations are used for the one-to-many model.

\subsection{Single Round Model}

In [SV05] the optimization objective is to maximize the expected number of sent messages during a single round. Therein the optimization should concern all numbers of senders from a given range $\left[n_{0}, N_{0}\right]$ and receivers from $\left[m_{0}, M_{0}\right]$ for the one-to-many model. Note that we count only messages that have been successfully transmitted and not the number of successfully received messages. Because of duplicates in the one-to-many model the number of received ones can be larger than the number of transmitted ones.

We will restate some of the results for maximizing

$$
\min _{n \in\left[n_{0}, N_{0}\right]} \min _{m \in\left[m_{0}, M_{0}\right]} \mathbf{E}\left[M_{p, p^{\prime}}^{1-\mathrm{m}}(n, m)\right] .
$$

For the expectation this is equivalent to minimize the following term:

Lemma 1 [SV05] The expectation of the number of successfully transmitted messages in the one-to-many model is

$\mathbf{E}\left[M_{p, p^{\prime}}^{1-m}(n, m)\right]=\sum_{i=1}^{C} n p_{i}\left(1-p_{i}\right)^{n-1}\left(1-\left(1-p_{i}^{\prime}\right)^{m}\right)$.

The one-to-one model is not considered in [SV05]. The corresponding lemma is:

Lemma 2 The expectation of the number of successfully transmitted messages in the one-to-one model is

$\mathbf{E}\left[M_{p, p^{\prime}}^{1-1}(n, m)\right]=\sum_{i=1}^{C} n p_{i}\left(1-p_{i}\right)^{n-1} m p_{i}^{\prime}\left(1-p_{i}^{\prime}\right)^{m-1}$.

The following trivial lower bound for the transmitted messages holds for $n, m \leq C$.

Lemma 3 In both communication models at most $\min \{m, n\}$ messages can be transmitted.

Nodes can select one of the following probability distributions for the channel selection:

\section{Definition 2}

1. Uniform distribution: $\quad p_{i}^{u n i}:=\frac{1}{C}$

2. Geometric distribution:

$$
p_{i}^{g e o}:= \begin{cases}\frac{1}{2^{i}}, & \text { if } i<C \\ \frac{1}{2^{C-1}}, & \text { if } i=C\end{cases}
$$

3. Factorized geometric distribution with the parameter $s \in \mathbb{N}$ uses the geometric distribution with $C / s$, assuming $C$ is a multiple of $s$.

$$
p_{i}^{s \text {-geo }}:=\frac{1}{s} p_{\left\lceil\frac{i}{s}\right\rceil}^{\text {geo }}=\frac{1}{s \cdot 2^{\left\lceil\frac{i}{s}\right\rceil}}
$$

Both senders and receivers can vary independently the probability distribution from round to round. Here, we consider that senders and receivers use the same one. It is an open question whether this is the best choice. However, intuitively it seems reasonable that all nodes should choose the same probability distribution. 


\subsection{Multiple Round Model}

In this paper we prove that a multiple round model can improve the number of transmitted messages considerably compared to any single round concept which is repeated for the same number of rounds. The reason is that in the multiple round model varying the used probability distribution in each round improves the performance.

In this model we have $T$ rounds. In each round senders and receivers use probability distributions for the channel selection: $P=\left(p_{1}, \ldots, p_{T}\right)$ for the senders and $P^{\prime}=$ $\left(p_{1}^{\prime}, \ldots, p_{T}^{\prime}\right)$ for the receivers. After each round all senders, having delivered a message in that round, drop out of the system. Analogous all receivers leave which have successfully received a message. This implies for the one-to-one case that if $n>m$ is valid, at least $n-m$ senders remain at the end. In the case of $m>n$ at least $m-n$ receivers will remain in the one-to-one model.

In the one-to-many model the situation is more complicated since the additional duplicated messages cause that more receivers drop out. Let $d$ be the number of duplicates over all rounds, then if $n+d>m$, at least $n+d-m$ senders remain at the end, and if $n+d<m$ then at least $m-d-n$ receivers remain.

In the multiple round model the same notations for the number of transmitted messages from definition 1 are used for the one-to-one and the one-to-many model. Formally, $M_{p_{i}, p_{i}^{\prime}}^{1-1}(n, m)$ or $M_{p_{i}, p_{i}^{\prime}}^{1-\mathrm{m}}(n, m)$ can be described as the outcome of a Markov process. We refer to this model for the analysis of our results in the following section.

\section{Results}

We start with the investigation of the uniform distribution. It is presented that this behaves very well in the single round scheme if the number of channels approximates the number of senders or the number receivers.

\subsection{The Uniform Distribution for the Sin- gle Round Scheme}

For the one-to-many communication model it was shown in [SV05]:

\section{Theorem 1 [SV05]}

$$
\begin{gathered}
\left(1-\frac{1}{e}\right) n e^{-\frac{n-1}{C-1}} \min \{1, p m\} \leq \mathbf{E}\left[M_{p^{\text {uni }}}^{1-m}(n, m)\right] \\
\leq n e^{-\frac{n-1}{C}} \min \{1, p m\}
\end{gathered}
$$

We now prove the analogous theorem for the one-to-one communication case.

\section{Theorem 2}

$$
\frac{n m}{C} \cdot e^{-\frac{n+m-2}{C-1}} \leq \mathbf{E}\left[M_{p^{u n i}}^{l-1}(n, m)\right] \leq \frac{n m}{C} \cdot e^{-\frac{n+m-2}{C}}
$$

Proof: From Lemma 2 we can conclude

$$
\begin{aligned}
& \mathbf{E}\left[M_{p}^{1-1}(n, m)\right] \\
& =\sum_{i=1}^{C} n p(1-p)^{n-1} m p(1-p)^{m-1} \\
& =\sum_{i=1}^{C} \frac{n}{C}\left(1-\frac{1}{C}\right)^{n-1} \frac{m}{C}\left(1-\frac{1}{C}\right)^{m-1} \\
& =\frac{n m}{C}\left(1-\frac{1}{C}\right)^{n-1}\left(1-\frac{1}{C}\right)^{m-1} \\
& \leq \frac{n m}{C} e^{-\frac{n+m-2}{C}}
\end{aligned}
$$

For a lower bound we get

$$
\begin{aligned}
\mathbf{E}\left[M_{p}^{1-1}(n, m)\right] & =\frac{n m}{C}\left(1-\frac{1}{C}\right)^{n-1}\left(1-\frac{1}{C}\right)^{m-1} \\
& \geq \frac{n m}{C} \cdot e^{-\frac{n+m-2}{C-1}}
\end{aligned}
$$

which follows from $\left(1-\frac{1}{n}\right)^{n} \leq \frac{1}{e} \leq\left(1-\frac{1}{n}\right)^{n-1}$ for all $n \geq 1$.

\subsection{A Reliable Multiple Round Scheme}

We now present the Funnel protocol which is described by a series of uniform probability distributions. Accordingly, we simplify $p^{\text {uni }}$ to $p$.

Definition 3 For a constant $\beta>1$ we define the set of probability distributions $p_{1}, \ldots, p_{T}$ as the $\beta$-Funnel distribution, where the probability to choose channel $i$ in round $t$ is $p_{t}:=\left\lceil C \beta^{1-t}\right\rceil$ and $T=\left\lceil\log _{\beta} C\right\rceil$.

Intuitively the protocol works as follows. It slowly reduces the number of channels until it approximates $\max \{n, m\}$. Then, the uniform probability distributions guarantees the delivery of $\Theta(\min \{n, m\})$ messages with constant probability. So some senders and receivers drop out because they have performed their task. Resultant, the next round continues with a reduced number of senders and receivers. For a good throughput also the number of channels continues to reduce. The process is done very carefully, since if this decrease is too fast, the number of channels is higher than the $\max \{n, m\}$. This continues until either no senders or receivers are left. Then, there is no chance that any message can be delivered and the residual probability distribution do not contribute to the success of the protocol.

So, for an appropriate choice of $\beta$ this protocol manages to deliver the optimal number of messages. 
Theorem 3 Let $\min \{m, n\} \geq C$. For any $\epsilon \in(0,1)$ and $\beta=1+\frac{1}{\left(\log \frac{1}{\epsilon}\right)(\log C)}$ the $\beta$-Funnel protocol delivers $\min \{n, m\}$ messages in $O\left(\left(\log \frac{1}{\epsilon}\right)(\log C)^{2}\right)$ rounds with probability $1-\epsilon$ in the one-to-one-model.

Proof: Consider the rounds $t \in\left[T_{0}, T_{1}\right]$ where $\frac{1}{2} \max \{n, m\} \leq C \beta^{1-t} \leq \max \{n, m\}$. We assume that no senders and receivers have been dropped out before (otherwise we consider a later point of time where this inequality holds).

From Theorem 2 it follows for the messages delivered in one round:

$\mathbf{E}\left[M_{p}^{1-1}(n, m)\right] \geq \frac{n m}{C \beta^{1-t}} \cdot e^{-\frac{n+m-2}{C \beta^{1-t}-1}} \geq \frac{1}{2 e^{2}} \min \{n, m\}$

Let $X:=\min \{n, m\}-M_{p}^{1-1}(n, m)$ denote the number of messages not delivered in this round. Then $\mathbf{E}[X] \leq\left(1-\frac{1}{2 e^{2}}\right) \min \{n, m\}$. From the Markov inequality $\operatorname{Prob}[X \geq k \mathbf{E}[X]] \leq \frac{1}{k}$ for all $k>1$ it follows:

$$
\begin{aligned}
& \text { Prob }\left[X \geq\left(1-\frac{1}{4 e^{2}}\right) \min \{n, m\}\right] \\
& \leq \text { Prob }\left[X \geq \frac{1-\frac{1}{4 e^{2}}}{1-\frac{1}{2 e^{2}}} \mathbf{E}[X]\right] \leq \frac{1-\frac{1}{2 e^{2}}}{1-\frac{1}{4 e^{2}}}=\frac{4 e^{2}-2}{4 e^{2}-1}
\end{aligned}
$$

So, there is a constant success probability $q=\frac{4 e^{2}-2}{4 e^{2}-1}$ for the case that at least $r \min \{n, m\}$ messages are delivered, whereas $r=\frac{1}{4 e^{2}}$. Now consider the following Markov process with states $S_{0}, S_{1}, \ldots, S_{\log _{\frac{1}{1-r}}} \min \{n, m\}$. State $S_{z}$ is active if at least $(1-r)^{z-1} \min \{n, m\}$ messages are not sent. We can now conclude for the transition probability from $S_{i} \rightarrow S_{i+1}$ being at least $q$. With the residual probability of at most $1-q$ the state $S_{i} \rightarrow S_{i}$ persists for one round. The expectation of the duration $D$ of this Markov process to end in state $\log _{\frac{1}{1-r}} \min \{n, m\}$ is therefore at least

$$
\mathbf{E}[D]=\frac{1}{1-q} \log _{\frac{1}{1-r}} \min \{n, m\}=\Theta(\log \min \{n, m\}) .
$$

Using the Chernoff bound for $\delta>0$ :

$$
\operatorname{Prob}[D \geq(1+\delta) \mathbf{E}[D]] \leq 2^{-\delta \mathbf{E}[D]} .
$$

it follows for $\delta \geq c \log C$ the error probability not being in state $q_{\log _{\frac{1}{1-r}} \min \{n, m\}}$, i.e. still having messages to be delivered, is at most $\epsilon \leq 2^{-\delta \mathbf{E}[D]}$. Solving this equation, we receive $\delta \geq \frac{-\log \epsilon}{E[D]}$. Hence, $\delta=-\log \epsilon$ is a valid choice for $\delta$.

Therefore, if we choose $\beta$ in such a way that the number of rounds is at least $\Theta\left(\log \frac{1}{\epsilon} \log C\right)$, in which $\frac{1}{2} \max \{n, m\} \leq$ $C \beta^{1-t} \leq \max \{n, m\}$ holds, then all messages are delivered with probability $1-\epsilon$. An appropriate choice of $\beta$ is

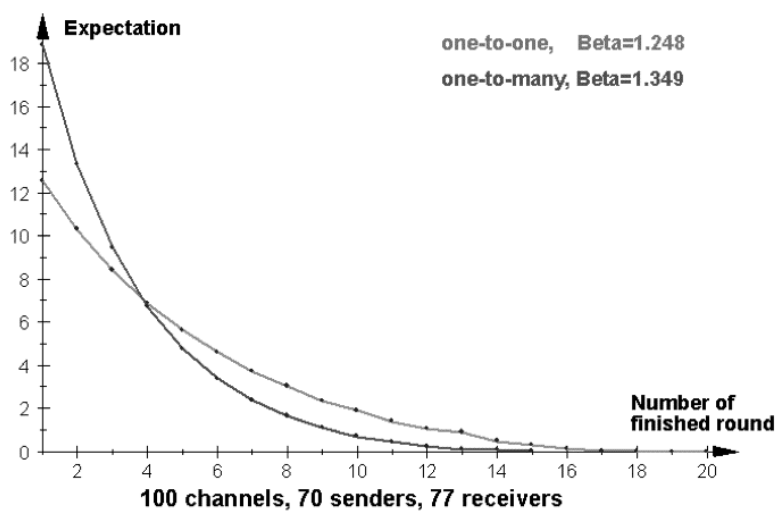

Figure 2. A choice of $\beta \in[1.2,1.4]$ suffices for delivering all messages

therefore $\beta=1+\frac{1}{\log \frac{1}{\epsilon} \log C}$, since $\max \{n, m\} \leq C$ and the number of rounds is bounded by $O\left(\log \frac{1}{\epsilon} \log ^{2} C\right)$.

From this theorem we can deduce the following corollory.

Corollary 1 There is an $O\left(\log ^{2} C\right)$-round oblivious probabilistic round protocol that delivers all messages in the oneto-one model and causes either all senders or all receivers to drop out in the one-to-many model with arbitrarily small constant error probability.

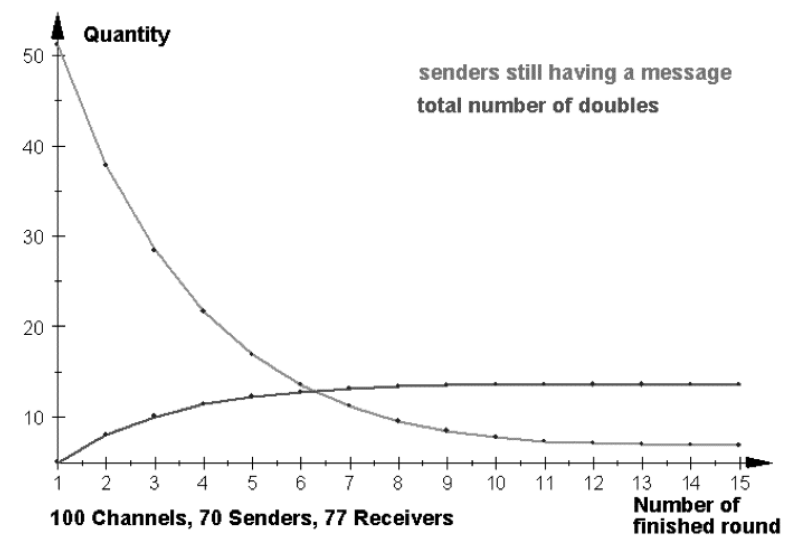

Figure 3. A typical source of transmission failure in the one-to-many model are message doubles.

The simulation results shown in Figure 2 show that a larger choice of $\beta$ can be used in practice, while constant factors are not particularly large. So, even for large scenarios with up to 100 nodes a constant factor of 1.25 works well and leads to up to 18 rounds of the Funnel protocol. 
In Figure 3 the situation for the one-to-many communication model is shown. The line starting at 50 describes the number of unfinished senders. The line starting at 0 shows the number of message doubles. Experiments show that a small choice of $\beta$ decreases the number of doubles. However, it goes along with an increase of the number of rounds of the Funnel protocol.

\subsection{An Upper Bound for the Single Round Model}

We now prove the limits of single round communication protocols. For this we show that there is an upper bound on the number of messages that can be transmitted within one round, if the number of senders and receivers is unknown.

Lemma 4 For constants $c=\left(1-\frac{1}{e}\right) \min _{i}\{1-p\}$ and $c^{\prime}=$ $e^{\max _{i}\{p\}}$ we have

$$
\begin{gathered}
c \sum_{i=1}^{C} H\left(\frac{(n-1) \cdot p}{1-p}\right) \cdot \min \{1, m p\} \\
\leq \mathbf{E}\left[M_{p}^{n, m}\right] \leq c^{\prime} \sum_{i=1}^{C} H(n \cdot p) \cdot \min \{1, m p\},
\end{gathered}
$$

where $H(x):=x e^{-x}$.

Proof: First note that for all $p \in[0,1], m>1$ :

$$
\left(1-\frac{1}{e}\right) \min \{1, p m\} \leq\left(1-(1-p)^{m}\right) \leq \min \{1, p m\}
$$

Further observe that $(1-p)^{n-1} \leq e^{-p n+p} \leq e^{-p n} e^{p}$ which implies the upper bound.

For the lower note that for all $n>1:\left(1-\frac{1}{n}\right)^{n-1} \geq \frac{1}{e}$ which implies $(1-p) \geq e^{-\frac{p}{1-p}}$. Therefore: $n p(1-p)^{n-1} \geq$ $n p e^{-\frac{n p}{1-p}} \geq(n-1) p e^{-\frac{n p}{1-p}}$. From this the lower bound follows straight-forward.

We investigate probability distributions $p$ with $0 \leq p_{i} \leq \frac{1}{2}$. So, $c \geq \frac{1}{2}\left(1-\frac{1}{e}\right) \approx 0.316$ and $c^{\prime} \leq e^{\frac{1}{2}} \approx 1.649$. For the analysis it is crucial to understand the meaning of the function $H(x)=x e^{x}$ visualized in Fig. 4. We will use the following Lemma.

\section{Lemma 5 It holds}

$$
\begin{aligned}
& \forall x \in\left[0, \frac{1}{2}\right]: \frac{1}{\sqrt{e}} x \leq x e^{-x} \leq x \\
& \forall x \in\left[\frac{1}{2}, 2\right]: \quad \frac{2}{e^{2}} \leq x e^{-x} \leq \frac{1}{e} \\
& \forall x \geq 2: 2 \cdot e^{-x} \leq x e^{-x} \leq 2 \cdot 2^{-x} \\
& \int_{x=0}^{\infty} x e^{-x} d x=1
\end{aligned}
$$

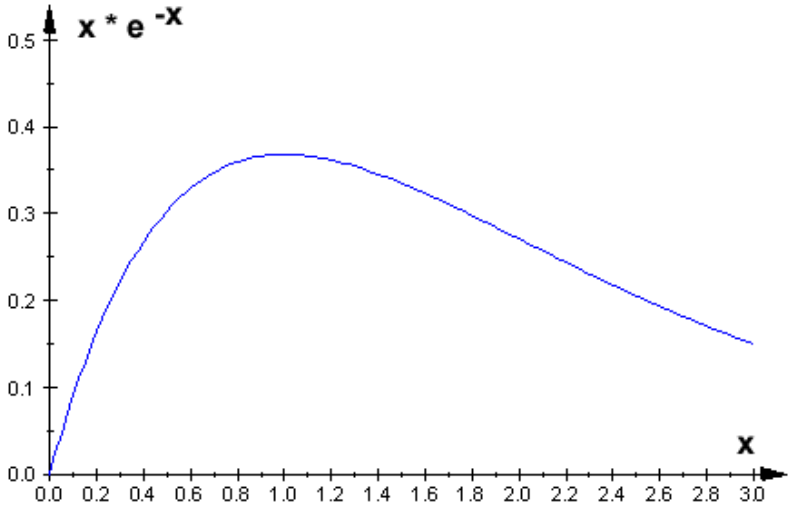

Figure 4. The function $x \mapsto x \cdot e^{-x}$

Proof: follows by applying straight-forward mathematical analysis.

Theorem 4 The factorized probability distribution is asymptotically optimal if the number of channels is unlimited, i.e. there exists $c>0$ for all $n_{0} \leq N_{0} \leq m_{0} \leq M_{0}$ such that for $s=\left\lfloor\frac{n_{0}}{2}\right\rfloor$

$$
\begin{gathered}
\min _{n \in\left[n_{0}, N_{0}\right]} \min _{m \in\left[m_{0}, M_{0}\right]} \mathbf{E}\left[M_{p^{s-g e o}, p^{s-g e o}}^{1-m}(n, m)\right] \\
\geq c \cdot \sup _{p, p^{\prime}} \min _{n \in\left[n_{0}, N_{0}\right]} \min _{m \in\left[m_{0}, M_{0}\right]} \mathbf{E}\left[M_{p_{i}, p_{i}^{\prime}}^{1-m}(n, m)\right] .
\end{gathered}
$$

The proof uses the following ideas.

\section{- Discretization of probabilities}

First, we show that the performance of any probability distribution can be approximated by a probability distribution that uses only weights of the form $p_{i}=\frac{1}{2^{j}}$ for some integers $j$. Furthermore, we show it suffices to consider number of channels being powers of 2 with only a constant factor loss of precision.

\section{- Re-formulation by counting probabilities}

Now every probability distribution can be described by the number of channels chosen with probability $2^{i}$, since channels are otherwise undistinguishable.

\section{- An upper bound on the probabilites}

Then, we present an upper bound by simplifying the optimization term. Combining this upper bound with the result of [SV05] completes the proof at the end of this subsection.

Lemma 6 The probability distribution $p$ maximizing the term

$$
\min _{\nu \in\left\{\log n_{0}, \cdots, \log N_{0}\right\}} \sum_{i=1}^{C} H\left(2^{\nu} p_{i}\right)
$$


gives a linear approximation of the optimal probability distributions $p, p^{\prime}$ maximizing the term

$$
\min _{n \in\left[n_{0}, N_{0}\right]} \min _{m \in\left[m_{0}, M_{0}\right]} \mathbf{E}\left[M_{p_{i}, p_{i}^{\prime}}^{n, m}\right]
$$

for $n_{0} \leq N_{0} \leq m_{0} \leq M_{0}$.

Proof: The term involving $p^{\prime}$ can be approximated by a constant factor since $m_{0} \geq N_{0}$. The rest follows by Lemma 4 and Lemma 5 which shows that rounding the number of senders to the next power of two decreases the term by at most a factor of two.

Lemma 7 Let

$T(p):=\min _{\nu \in\left\{\log n_{0}, \cdots, \log N_{0}\right\}} \sum_{i=1}^{C} H\left(2^{\nu} p_{i}\right)$. For every probability distribution $p$ there exists a probability distribution $\phi$ such that for all $i$ the probability $\phi_{i}$ to choose channel $i$ is a power of 2 , i.e. $\phi_{i} \in\left\{2^{-1}, 2^{-2}, \ldots,\right\}$, such that

$$
T(p) \leq 2 T(\phi) \leq 2 T(p) .
$$

Proof: Round all probabilities to the next lower interval limit. From Lemma 5 it follows that this decreases the term by at most a factor of 2 .

Now we describe this modified probability distribution by the vector $X(p)=\left\{x_{1}, x_{2}, \ldots,\right\}$ where $x_{i}$ counts the channels chosen with probability $2^{-i}$, i.e.

$$
x_{j}:=\left|\left\{i: p_{i}=2^{i}\right\}\right| .
$$

Since $p$ is a probability distribution we have

$$
\sum_{j=1}^{\infty} x_{j} 2^{-j}=1
$$

The maximization goal $T(p)$ can be rewritten to

$$
\begin{aligned}
T(X(p)) & :=\min _{\nu \in\left\{\log n_{0}, \ldots, \log N_{0}\right\}} \sum_{j=1}^{\infty} x_{j} H\left(2^{\nu-j}\right) \\
& =\min _{\nu \in\left\{\log n_{0}, \ldots, \log N_{0}\right\}} \sum_{i=1}^{C} H\left(2^{\nu} p_{i}\right)=T(p)
\end{aligned}
$$

We now use the following Lemma.

Lemma 8 For all $\nu \geq 1$, for all $x_{1}, x_{2}, \ldots$ with $\sum_{j=1}^{\infty} x_{j} 2^{-j} \leq 1$ we have

$$
\sum_{j=1}^{\infty} x_{j} H\left(2^{\nu-j}\right) \leq 3 \cdot 2^{\nu}
$$

Proof: From Lemma 5 (3) it follows for the sum

$$
\sum_{j=1}^{\nu-1} x_{j} H\left(2^{\nu-j}\right) \leq \sum_{j=1}^{\nu-1} 2 \cdot x_{j} 2^{-2^{\nu-j}} \leq \sum_{j=1}^{\nu-1} 2 \cdot x_{j} 2^{\nu-j} \leq 2^{\nu+1} .
$$

From Lemma 5 (1) and (2) it follows for the sum

$$
\sum_{j=\nu-1}^{\infty} x_{j} H\left(2^{\nu-j}\right) \leq \sum_{j=\nu-1}^{\infty} x_{j} 2^{\nu-j} \leq 2^{\nu} .
$$

All this implies for all $p$ that $T(X(p))=O\left(n_{0}\right)$. On the other hand it was proved in [SV05] the corresponding upper bound.

Theorem 5 [SV05] Factorized geometric distribution: Let $s \leq n$ and $C \geq s \log n$ :

$$
\mathbf{E}\left[M_{p^{s-g e o}, p^{s-g e o}}^{l-m}(n, m)\right]= \begin{cases}\Theta\left(s \frac{m}{n}\right), & \text { if } m \leq n \\ \Theta(s), & \text { if } m \geq n\end{cases}
$$

Since in our case $m \geq n$ this completes the proof of Theorem 4.

\section{Conclusions}

We presented a process for the channel utilization over multiple rounds. It bases on the uniform distribution and can serve as a MAC protocol for sensor networks if many channels are available and little information needs to be timely distributed. In such scenarios the handshake on a control channel may lead to a communication bottleneck. With the analytical proven upper bound of $O\left(\log ^{2} n\right)$ rounds which in practice can be reduced to some 20 rounds, the Funnel protocol utilizes the inherent parallelism of the independent channels. The communication overhead to a pseudo-random communication channel utilization scheme is bounded by a polylogarithmic number of channels. Since the communication flow is unpredictable and the specific sets of senders and receivers change within time, this protocol forms a simple and elegant alternative to state-of-the-art communication protocols.

The Funnel protocol can be combined with several types of routing protocols. The best fit for this MAC protocol is one using parallel communication between variable sets of senders and receivers. For this, one can consider a hop based zoning protocol as used by $1 \mathrm{QK}\left[\mathrm{SJR}^{+} 04\right]$. For delivering sensor data in a super-round, $t-d$ senders are nodes with data in hop distance $d+1$ from the communication sinks and receivers are nodes with empty buffer space in hop distance $d$. A super-round consists of the $O\left(\log ^{2} n\right)$ rounds of the described MAC protocol. This leads to a simple, robust, fast, and novel sensor networking protocol. Of course, additional mechanisms for synchronization and zoning need to be adopted.

Another implication of this paper is that a combination of different probability distributions in a multiple round model outperforms choosing optimal single round oblivious probability distributions. For this we have proved an upper 
bound on the transmitted messages for the single round channel utilization protocol if the number of senders and receivers is unknown. This upper bound solves the recently stated open problem by the authors of this paper in [SV05]. The conclusion is that the proposed factorized geometric probability distribution for channel utilization in the single round model in [SV05] is in fact asymptotically optimal. Yet, it is outperformed by a multiple round protocol like the Funnel protocol.

\section{References}

[AHR04a] Baruch Awerbuch, David Holmer, and Herbert Rubens. The Pulse Protocol: Energy Efficient Infrastructure Access. IEEE Infocom, 2004.

[AHR ${ }^{+}$04b] Baruch Awerbuch, David Holmer, Herbert Rubens, I.-J. Wang, and Kirk Chang. The Pulse Protocol: Sensor Network Routing and Power Saving. MILCOM, 2004.

[BMT04] Philipp Blum, Lennart Meier, and Lothar Thiele. Improved interval-based clock synchronization in sensor networks. In IPSN'04: Proceedings of the third international symposium on Information processing in sensor networks, pages 349-358, New York, NY, USA, 2004. ACM Press.

[BZ97] Flaminio Borgonovo and Michele Zorzi. Slotted aloha and cdpa: a comparison of channel access performance in cellular systems. Wirel. Netw., 3(1):43-51, 1997.

[EB04] Cheng Tien Ee and Ruzena Bajcsy. Congestion control and fairness for many-to-one routing in sensor networks. In SenSys '04: Proceedings of the 2nd international conference on Embedded networked sensor systems, pages 148-161, New York, NY, USA, 2004. ACM Press.

[EGE02] Jeremy Elson, Lewis Girod, and Deborah Estrin. Fine-grained network time synchronization using reference broadcasts. SIGOPS Oper. Syst. Rev., 36(SI):147-163, 2002.

[GKS03] Saurabh Ganeriwal, Ram Kumar, and Mani B. Srivastava. Timing-sync protocol for sensor networks. In SenSys '03: Proceedings of the 1st international conference on Embedded networked sensor systems, pages 138-149, New York, NY, USA, 2003. ACM Press.
[Gol04] N. Golmie. Bluetooth dynamic scheduling and interference mitigation. Mob. Netw. Appl., 9(1):21-31, 2004.

[Gro99] Bluetooth Special Interest Group. Specifications of the Bluetooth System Vol. 1, v. 1.OB 'Core' and Vol.2, v. 1.OB 'Profiles'. December 1999.

[HCB00] Wendi Rabiner Heinzelman, Anantha Chandrakasan, and Hari Balakrishnan. Energyefficient communication protocol for wireless microsensor networks. In HICSS '00: Proceedings of the 33rd Hawaii International Conference on System Sciences-Volume 8, page 8020, Washington, DC, USA, 2000. IEEE Computer Society.

[HGT04] Matthias Handy, Frank Grassert, and Dirk Timmermann. Dcp: A new data collection protocol for bluetooth-based sensor networks. In DSD '04: Proceedings of the Digital System Design, EUROMICRO Systems on (DSD’04), pages 566-573, Washington, DC, USA, 2004. IEEE Computer Society.

[HLMV05] Qi Han, Iosif Lazaridis, Sharad Mehrotra, and Nalini Venkatasubramanian. Sensor data collection with expected reliability guarantees. In PERCOMW'05: Proceedings of the Third IEEE International Conference on Pervasive Computing and Communications Workshops (PERCOMW'05), pages 374-378, Washington, DC, USA, 2005. IEEE Computer Society.

$\left[\mathrm{HSW}^{+}\right.$00] Jason Hill, Robert Szewczyk, Alec Woo, Seth Hollar, David Culler, and Kristofer Pister. System architecture directions for networked sensors. In ASPLOS-IX: Proceedings of the ninth international conference on Architectural support for programming languages and operating systems, pages 93-104, New York, NY, USA, 2000. ACM Press.

[IGE00] Chalermek Intanagonwiwat, Ramesh Govindan, and Deborah Estrin. Directed diffusion: a scalable and robust communication paradigm for sensor networks. In MobiCom '00: Proceedings of the 6th annual international conference on Mobile computing and networking, pages 56-67, New York, NY, USA, 2000. ACM Press.

[JE03] Jaein Jeong and Cheng Tien Ee. Forward Error Correction in Sensor Networks. $U C B$ Technical Report, May 2003. 
[Kar90] P. Karn. MACA- a New Channel Access Method for Packet Radio. ARRL/CRRL Amateur Radio 9th Computer Networking Conference, pages 134-140, 1990.

[KKIK03] Rajgopal Kannan, Ram Kalidindi, S. S. Iyengar, and Vijay Kumar. Energy and rate based mac protocol for wireless sensor networks. SIGMOD Rec., 32(4):60-65, 2003.

[LH05] K. Langendoen and G. Halkes. Embedded System Handbook, R. Zurawski (editor). CRC Press, 2005. Chapter: Energy-efficient Medium Access Control.

[Nam84] C. Namislo. Analysis of mobile radio slotted ALOHA networks. IEEE J. Selected Areas Commun. 2, 199-204, August 1984.

[PHC04] Joseph Polastre, Jason Hill, and David Culler. Versatile low power media access for wireless sensor networks. In SenSys '04: Proceedings of the 2nd international conference on Embedded networked sensor systems, pages 95107, New York, NY, USA, 2004. ACM Press.

[Rob75] Lawrence G. Roberts. Aloha packet system with and without slots and capture. SIGCOMM Comput. Commun. Rev., 5(2):28-42, 1975.

[ROGLA03] Venkatesh Rajendran, Katia Obraczka, and J. J. Garcia-Luna-Aceves. Energy-efficient collision-free medium access control for wireless sensor networks. In SenSys '03: Proceedings of the 1st international conference on Embedded networked sensor systems, pages 181-192, New York, NY, USA, 2003. ACM Press.

[Sch00] Jochen Schiller. Mobile Communications, Pages 37-42 and 61-81. 2000.

$\left[\mathrm{SCI}^{+}\right.$01] Eugene Shih, SeongHwan Cho, Nathan Ickes, Rex Min, Amit Sinha, Alice Wang, and Anantha Chandrakasan. Physical Layer Driven Protocol and Algorithm Design for EnergyEfficient Wireless Sensor Networks. International Conference on Mobile Computing and Networking, Proceedings of the 7th annual international conference on Mobile computing and networking, 272-287, 2001.

[SJR ${ }^{+}$04] Christian Schindelhauer, Michelle Liu Jing, Stefan Ruehrup, Klaus Volbert, Martin Dierkes, Andreas Bellgardt, Ruediger Ibers, and Ulrich Hilleringmann. Sensor Networks with more Features using less hardware. GOR International Conference Operations Research, Page 63, September 2004.

[SR98] Suresh Singh and C.S. Raghavendra. PAMAS - Power Aware Multi-Access protocol with Signalling for Ad Hoc Networks. SIGCOMM Comput. Commun. Rev., 28(3):5-26, 1998.

[SR03] Frank Siegemund and Michael Rohs. Rendezvous layer protocols for bluetooth-enabled smart devices. Personal Ubiquitous Comput., 7(2):91-101, 2003.

[SV05] Christian Schindelhauer and Kerstin Voß. Probability distributions for channel utilisation. In ADHOCNOW 2005: 4th International Conference on Ad-Hoc Networks \& Wireless, pages 271-284, October 2005.

[VL90] C. Vanderplas and J. P. M. Linnartz. Stability of mobile slotted ALOHA network with Rayleigh fading, shadowing and near-far effect. IEEE Trans. Vehic. Technol. 39, 359366, November 1990.

[WLLP01] Brett Warneke, Matt Last, Brian Liebowitz, and Kristofer S. J. Pister. Smart dust: Communicating with a cubic-millimeter computer. Computer, 34(1):44-51, 2001.

[YHE04] Wei Ye, John Heidemann, and Deborah Estrin. Medium access control with coordinated adaptive sleeping for wireless sensor networks. IEEE/ACM Trans. Netw., 12(3):493506, 2004.

[ZRS05] Tao Zheng, Sridhar Radhakrishnan, and Venkatesh Sarangan. Pmac: An adaptive energy-efficient mac protocol for wireless sensor networks. In IPDPS '05: Proceedings of the 19th IEEE International Parallel and Distributed Processing Symposium (IPDPS'05) - Workshop 12, page 237.1, Washington, DC, USA, 2005. IEEE Computer Society. 